\title{
MECHANISM OF ELECTROLYTE IMBALANCE \\ FOLLOWING UROINTESTINAL ANASTOMOSIS
}

\author{
STEPHEN A. KOFF, M.D. \\ From the Section of Urology, Department of Surgery, \\ University of Michigan Medical Center, \\ Ann Arbor, Michigan
}

\begin{abstract}
A case is reported of profound hyperchloremic acidosis and hypokalemic paralysis developing in a thirteen-year-old girl after cutaneous ileocystostomy. An in vivo study of the absorptive characteristics of the ileal segment clarified the pathogenesis of this syndrome and effectively guided further management.
\end{abstract}

The syndrome of hyperchloremic acidosis occurs with predictable frequency following ureterosigmoidostomy. The majority of patients demonstrate only mild electrolyte disturbances and often require no therapy. Severe electrolyte derangements can occur in those patients with underlying renal disease. Electrolyte imbalance following ileal urinary diversion, however, is unusual. Rapid urine transit and various mucosal absorptive characteristics may be responsible for the reduced incidence of this complication. A case of profound acidosis with hypokalemic paralysis occurring after ileal segment urinary diversion was recently observed. After clinical recovery in vivo study of the absorptive characteristics of the ileal segment clarified the pathogenesis of the syndrome and effectively guided further management.

\section{Case Report}

In 1967 a six-year-old myelodysplastic girl was seen with total urinary incontinence, chronic pyelonephritis, and renal calculi. After failure of multiple attempts to stabilize her urinary tract and to control incontinence, a urinary diversion with urethral closure was performed on March 15 , 1973. A 22-cm. segment of distal ileum was used to create a cutaneous ileocystostomy (Fig. 1A). Pre- and postoperative renal function studies and electrolytes were relatively normal (Table I). The patient was discharged after ten days, having had an uneventful postoperative course.
Within six weeks she was readmitted in a comatose state. For ten days prior to admission the patient had complained of generalized myalgia, progressive anorexia, and lethargy. Weakness of the arms occurred, and she had difficulty feeding herself. One day prior to admission, urine output diminished noticeably, and breathing became difficult. On the day of admission the patient was unable to be aroused and grand mal seizures developed en route to the medical center. There was no history of vomiting, diarrhea, fever, or upper respiratory illness.

In the emergency suite the patient appeared unresponsive and was intubated. On physical examination, blood pressure was $130 / 80 \mathrm{~mm}$. $\mathrm{Hg}$, pulse 110 and regular, temperature $97^{\circ} \mathrm{F}$., and respirations were assisted at 26 per minute. She was dehydrated. Pupils were fixed and dilated. There was no papilledema and the neck was supple. Chest and abdominal examinations were unremarkable except for healed surgical scars. The ileal stoma readily admitted an index finger. Deep tendon reflexes were absent; extremities were flaccid.

Admission laboratory data revealed: sodium $126 \mathrm{mEq}$., potassium $1.3 \mathrm{mEq}$., chloride 95 $\mathrm{mEq}$., and bicarbonate $5 \mathrm{mEq}$. per liter (Table I). Arterial blood gases were $\mathrm{pH} 6.84$, carbon dioxide pressure $30 \mathrm{~mm}$. $\mathrm{Hg}$, and oxygen tension 91 $\mathrm{mm}$. Hg. Hemogram revealed hematocrit 32.5, white blood count 36,300 cells per cubic millimeter; blood urea nitrogen $171 \mathrm{mg}$., serum creatinine $4.7 \mathrm{mg}$., glucose $207 \mathrm{mg}$, , and calcium 6.9 
TABLE I. Changes in serum electrolytes, renal function studies, and arterial blood gases before and after therapy

\begin{tabular}{|c|c|c|c|c|c|c|c|c|c|c|}
\hline DATE ( 1973) & $3 / 5$ & $3 / 15$ & $3 / 19$ & $\begin{array}{c}4 / 15 \\
8 \text { p. }\end{array}$ & $4 / 15$ & $\begin{array}{c}4 / 16 \\
1 \text { a. }\end{array}$ & $\begin{array}{c}4 / 16 \\
1 \mathrm{p} .\end{array}$ & $\begin{array}{l}4 / 16 \\
10 \mathrm{p} .\end{array}$ & $4 / 21$ & $7 / 31$ \\
\hline $\mathrm{Na}(\mathrm{mEq} / \mathrm{L})$ & 136 & i & 142 & 126 & : & 135 & $\vdots$ & 138 & 140 & 138 \\
\hline $\mathrm{K}(\mathrm{mEq} / \mathrm{L})$ & 3.8 & לे & 4.6 & 1.3 & & 3.8 & हે & 4. 1 & 3.7 & 4.2 \\
\hline $\mathrm{Cl}(\mathrm{mEq} / \mathrm{L})$ & 96 & $\frac{n}{0}$ & 1.07 & 95 & $\frac{i}{n}$ & 116 & 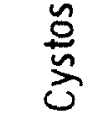 & 109 & 97 & 107 \\
\hline $\mathrm{HCO}_{3}(\mathrm{mEq} / \mathrm{L})$ & 26 & $\underline{E}$ & 23 & 5 & $\frac{\lambda}{\frac{\pi}{\pi}}$ & 6 & $\frac{u}{3}$ & 13.5 & 20 & 20 \\
\hline BUN $(m g \%)$ & 13 & 总 & 16 & 171 & Е્' & & $\frac{0}{\frac{0}{2}}$ & 160 & 64 & 22 \\
\hline Creat. ( $\mathrm{mg} \%$ ) & 0.3 & $\stackrel{\frac{\pi}{3}}{3}$ & 1.3 & 4.7 & 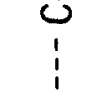 & & $n$ & 4.8 & 2.0 & 0.6 \\
\hline Arterial pH & & $i$ & & 6.84 & i & 7.24 & 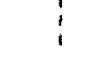 & 7.45 & & \\
\hline
\end{tabular}
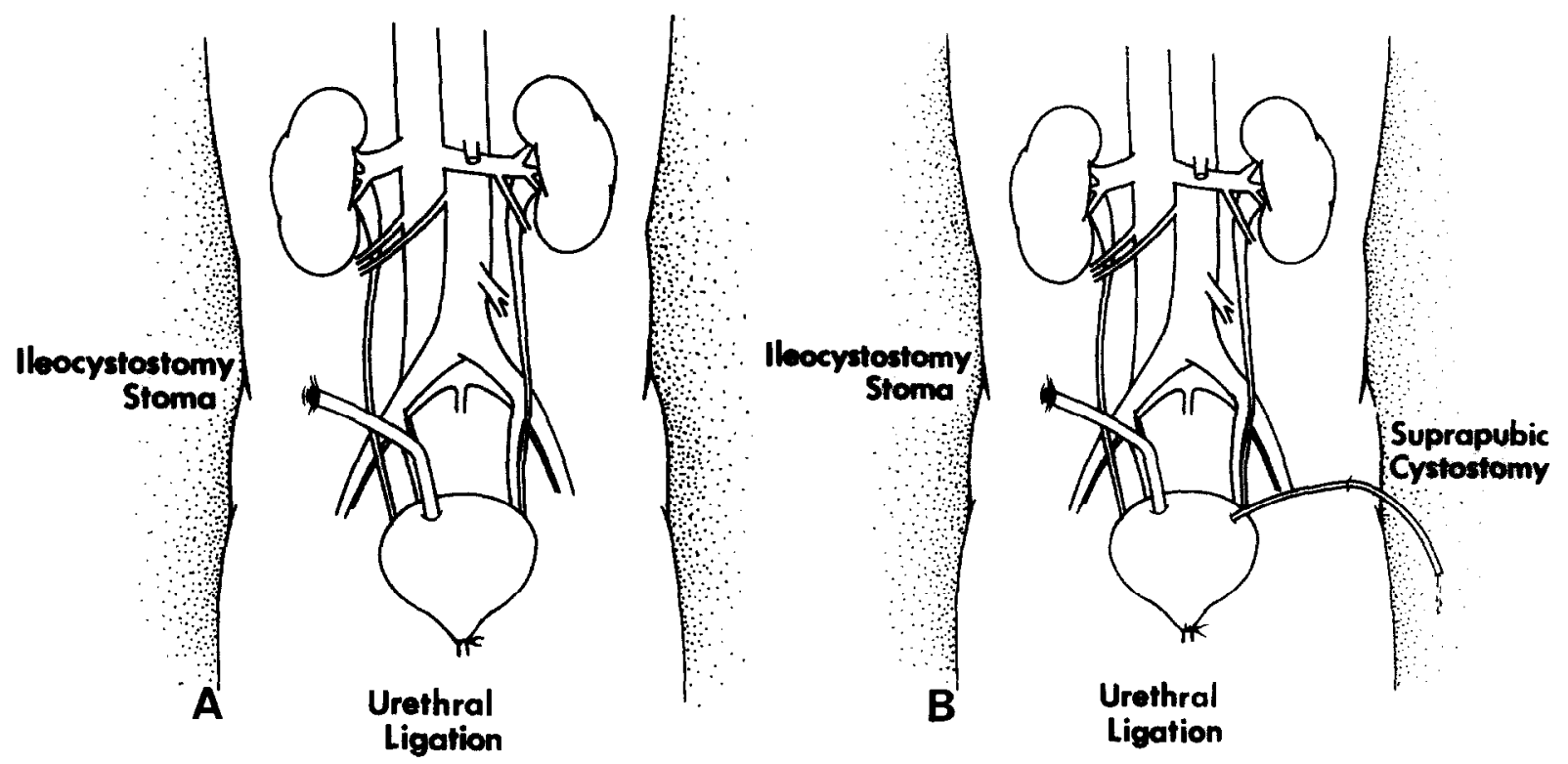

FIGURE 1. Schematic representation of patient's urinary tract following (A) urethral ligation and cutaneous ileocystostomy, and (B) suprapubic cystostomy.

mg. per $100 \mathrm{ml}$. Urinalysis revealed a $\mathrm{pH}$ of 7 , specific gravity 1.024 , no protein or sugar, and few white and red blood cells per high-power field.

Therapy was initiated with an intravenous infusion of $500 \mathrm{ml}$. 5 per cent dextrose and saline to which potassium chloride and sodium bicarbonate were added. After five hours the patient had received $176 \mathrm{mEq}$. sodium bicarbonate and
$160 \mathrm{mEq}$. potassium chloride. Urine output was 40 to $50 \mathrm{ml}$. per hour. Seizure activity was abolished, and the patient responded to simple commands. Laboratory studies had improved by April 16 (Table I).

An intravenous pyelogram and retrograde injection of the ileal loop were performed, and there was no evidence of obstruction. The loop, however, was nearly twice as long as was recorded 


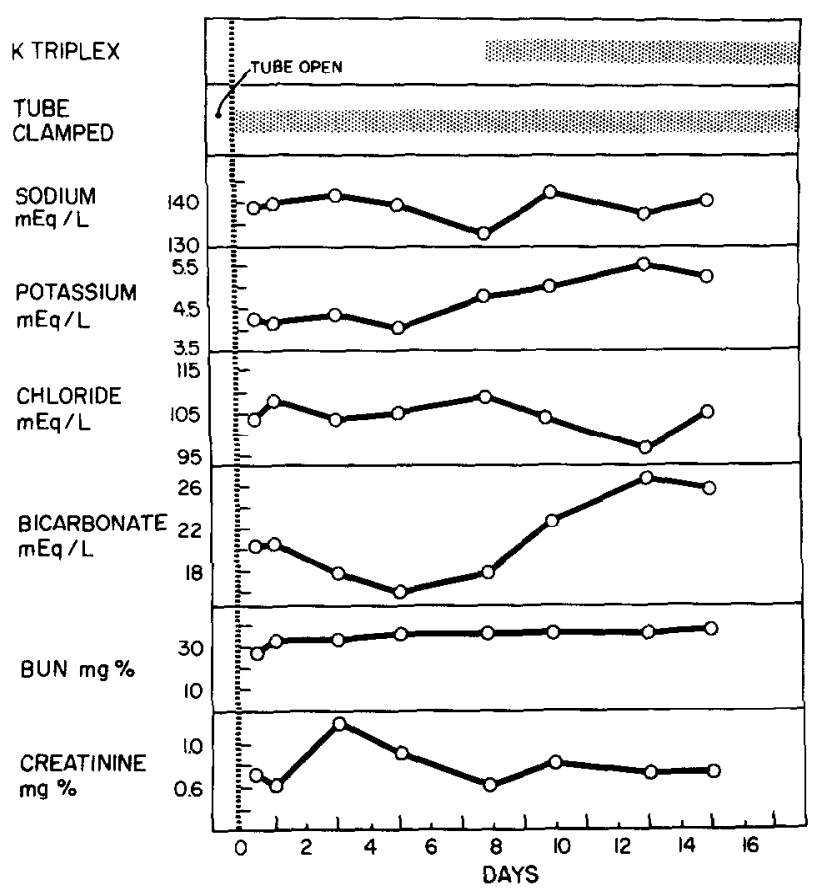

FIGURE 2. Changes in the levels of serum electrolytes and renal function studies related to clamping suprapubic cystostomy tube and to alkaline potassium therapy.

at the time of surgical intervention. A suprapubic cystostomy was performed to bypass the ileal segment (Fig. 1B). After twenty-four hours of therapy the patient was extubated and began talking. Total bicarbonate administered during this period was $330 \mathrm{mEq}$; total potassium chloride was $225 \mathrm{mEq}$. With the suprapubic tube draining, the patient made a rapid clinical and metabolic recovery. Renal function also returned to near normal levels by July 31 (Table I).

Several months later, after complete recovery, the pathogenesis of the ileal segment-induced metabolic derangement was studied. On August 1,1973 , the suprapubic tube was clamped, and all urine egressing from the ileal stoma was collected. The suprapubic tube was then partially opened to permit drainage of small amounts of urine continually through each twenty-four hour collection period. This suprapubic drainage represented primarily bladder urine before entrance into the ileal segment. All twenty-four-hour urine specimens were analyzed for electrolytes, urea nitrogen, and creatinine. Serum electrolyte and renal function determinations were obtained frequently (Fig. 2). The study was continued for sixteen days (Fig. 3A to D). On the eighth day, after the patient's serum bicarbonate dropped to $16 \mathrm{mEq}$. per liter, alkaline potassium supplement was begun (potassium acetate, citrate, and bi- carbonate representing $54 \mathrm{mEq}$. per day of potassium and bicarbonate). At the conclusion of the study, the patient, having experienced no untoward effects, was discharged with the suprapubic tube draining. She was readmitted one month later for revision of the ileal loop, at which time the segment was shortened to $16 \mathrm{~cm}$. and the suprapubic tube removed. Six months postoperatively she had normal renal function studies and electrolytes and was doing well without medication.

\section{Comment}

The syndrome of hyperchloremic acidosis with hypokalemic paralysis in association with ureterosigmoidostomy has been well described. ${ }^{1}$ Ileal segment urinary diversion is a rare cause of this syndrome; to my knowledge only once previously has so severe a metabolic disturbance been reported after ileal diversion. ${ }^{2}$ Ideally a short ileal segment will function as a conduit rather than as a reservoir. This will minimize the contact time between ileal mucosa and urine and will, therefore, reduce intestinal absorption. It has been suggested that the differences in absorptive characteristics between ileal and colonic mucosa accounts for the lower frequency of electrolyte aberrations. ${ }^{3}$ As impaired renal function contributes to the development and severity of this syndrome,${ }^{4}$ the reduced chance of hydronephrosis and chronic pyelonephritis developing after ileal diversion may also decrease the incidence of electrolyte disturbance. ${ }^{5}$

This case demonstrates the occurrence of profound acidosis and hypokalemic paralysis soon after cutaneous ileocystostomy. At the time of admission the diagnosis of hyperchloremic acidosis was not apparent from the initial laboratory determinations (Table I). Although a relative hyperchloremia existed, it was not until resuscitative efforts were underway that the more classic electrolyte pattern emerged. Therapy was directed toward the correction of dehydration and massive potassium and bicarbonate deficits. One cannot overemphasize the risks of combined potassium and bicarbonate replacement to patients with severe hypokalemic acidosis. Overzealous potassium repletion may exacerbate systemic acidosis by causing an intra- to extracellular hydrogen ion migration and may be lethal. Excessive bicarbonate replacement may effect an intracellular potassium shift with worsening of hypokalemia and associated neuromuscular impairment. Both ions must, therefore, be replaced concommitantly, and with careful clinical and 

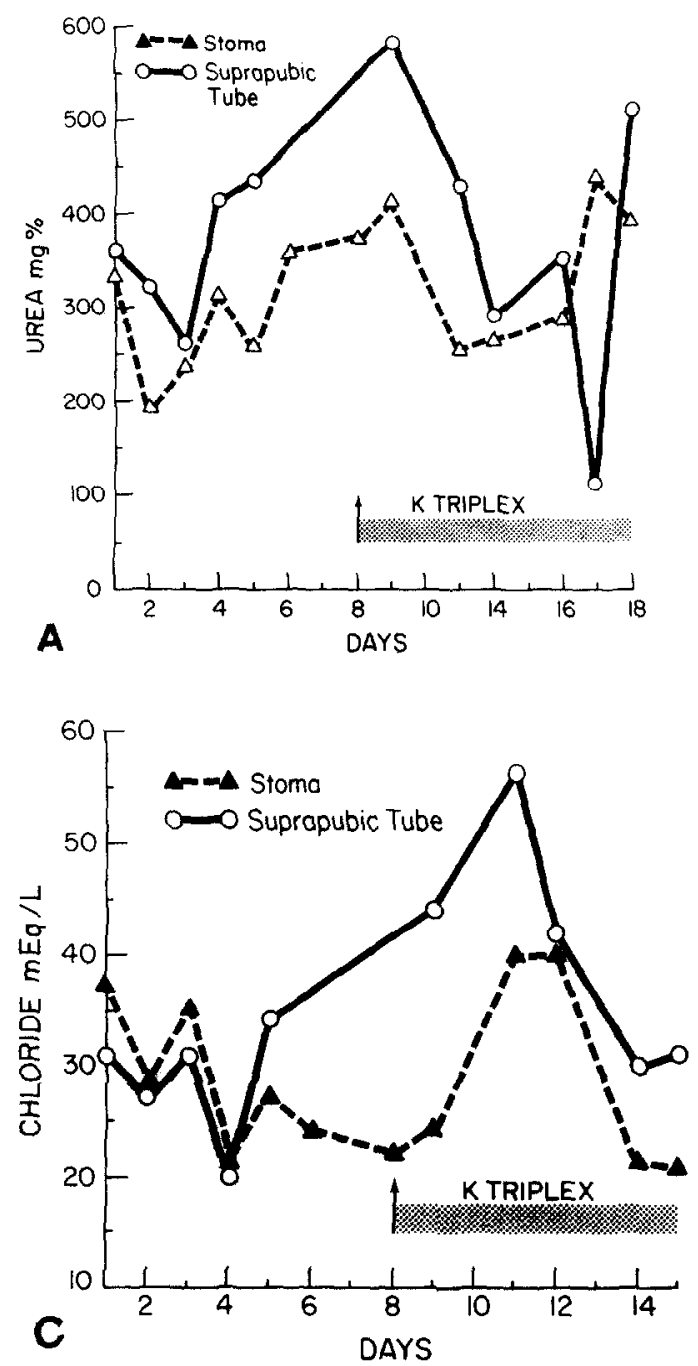
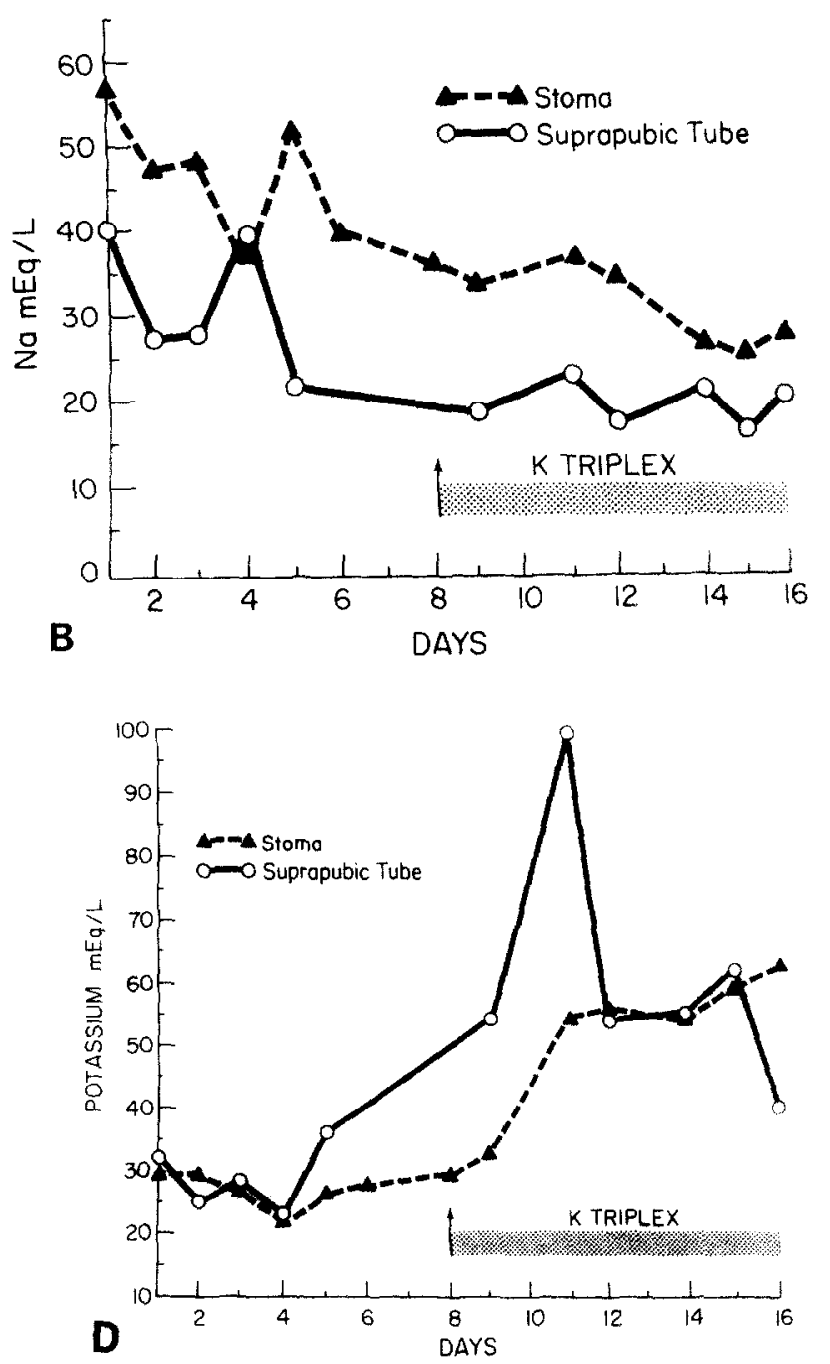

FIGURE 3. Comparisons of twenty-four-hour urine specimens obtained from ileocystostomy stoma with suprapubic cystostomy. (A) Urea concentration; (B) sodium concentration; (C) chloride concentration; and (D) potassium concentration.

laboratory monitoring, especially when large deficits exist. ${ }^{2,6}$

Soon after admission, contrast studies were performed which demonstrated a lengthy ileal segment without obstruction. A suprapubic eystostomy was performed to permit urine to bypass the ileal segment and, following this, deranged electrolyte and renal function studies rapidly approached normal. The presence of this cystostomy tube provided an opportunity to sample urine before presumably having the ileal mucosa alter its electrolyte composition. Comparison of the electrolyte content of urine before and after contact with the ileal segment clarified the mechanism which produced this patient's syndrome and demonstrated that clinically significant differences exist between the absorptive characteristics of the ileum and the colon.
Figure $3 \mathrm{~A}$ shows that the ileal segment reduced the urea concentration of bladder urine by 30 to $150 \mathrm{mg}$. per $100 \mathrm{ml}$. The patient constantly absorbed urea from the loop, and an osmotic diuresis developed in which urinary output was equal to or greater than intake, and bladder urine specific gravity was between 1.010 and 1.016 . Unlike the situation following ureterosigmoidostomy, however, in which sodium is readily absorbed from the colon and contributes to an osmotic diuresis, the ileal segment actually increased the sodium concentration of the urine passing through it and provided an avenue of continued sodium loss (Fig. 3B).

Figure 3C indicates that chloride was absorbed from the urine by the ileal loop. Although this absorption contributed to the osmotic load and diuresis, no appreciable elevation in serum 
chloride was noted during the study (Fig. 2). Madsen $^{8}$ has shown experimentally that active absorption of chloride by the ileal segment is associated with secretion of bicarbonate into the intestinal lumen. On the basis of partial inhibition of both chloride absorption and bicarbonate secretion with carbonic anhydrase inhibitors, he concluded that direct ionic exchange between bicarbonate and chloride existed and was etiologic in hyperchloremic acidosis. Examination of Figure 2 shows that serum bicarbonate was reduced from 22 to $16 \mathrm{mEq}$. per liter during the first five days of the study. No significant difference in chloride concentration developed between loop and bladder urine, however, during this same period (Fig. 3C). This suggests that although bicarbonate secretion and chloride absorption may at times occur concurrently, they are independent processes.

Ileal bicarbonate secretion appears to have produced the acidosis. Although urine bicarbonate was not measured directly, the ileal segment constantly alkalinized bladder urine. The $\mathrm{pH}$ of ileal stoma urine was measured throughout the study and found to be greater than 6.95. Simultaneous spot measurements of bladder urine $\mathrm{pH}$ through the suprapubic cystostomy ranged between 5 and 6 . On the basis of this low bladder urine $\mathrm{pH}$, renal handling of acid was believed to be appropriate. The magnitude and significance of this bicarbonate loss is seen in the reduction of serum bicarbonate from 22 to 16 $\mathrm{mEq}$. per liter during the first five days of the study (Fig. 2).

Hypokalemia occurs occasionally following ureterosigmoidostomy. Potassium loss is frequent but serum levels do not often reflect the extent of total body potassium depletion. ${ }^{7}$ Some authors believe that colonic potassium secretion plays a significant role in the development of hypokalemia. ${ }^{6}$ Madsen $^{8}$ has shown that potassium may be absorbed from isolated segments of ileum and colon. Equilibration generally occurs when the intraluminal potassium concentration is in the 10 to $30 \mathrm{mEq}$. per liter range. ${ }^{8}$

In our patient no ileal segment potassium secretion occurred. Bladder and ileal urine potassium concentrations were similar except during potassium supplementation when absorption of potassium by the ileum occurred (Fig. 3D). These findings suggest that hypokalemia following ileal urinary diversion is not due to intraluminal secretion of potassium.

Experimental urea diuresis actually produces secretion of potassium by the renal tubules to such an extent that potassium clearance may frequently exceed the actual amount filtered. ${ }^{9}$ Such a urea diuresis occurred continually in this patient and appears to be the mechanism by which profound hypokalemia developed. Figure $3 D$ confirms this by showing that during potassium replacement therapy, large amounts of potassium are still being excreted in the urine.

From this information we must conclude that the syndrome of hyperchloremic acidosis with hypokalemic paralysis in our patient resulted from too long an absorptive surface of ileal conduit. Despite apparently normal renal function studies, intrinsic renal parenchymal disease was an important contributing factor. The ileal segment actively absorbed chloride and urea and effected a sustained osmotic diuresis. Bicarbonate secretion rapidly caused acidemia by overwhelming the renal mechanisms for hydrogen ion secretion. Sodium loss contributed to dehydration and volume depletion which produced prerenal azotemia and further impaired renal homeostatic efforts. Potassium loss occurred passively and continually in association with osmotic diuresis; no active excretion of potassium could be demonstrated.

Supplementation of potassium and alkali was arbitrarily started when serum bicarbonate was reduced to $16 \mathrm{mEq}$. per liter. This therapy was successful in correcting bicarbonate levels and maintaining a normal serum potassium. Unfortunately, relentless urea and chloride absorption occurred unabated, and in- and output measurements reflected continued osmotic diuresis. Because of the rapidity with which the syndrome of acidosis and hypokalemia occurred and because the basic pathogenesis was unalterable by medical therapy, we elected to shorten the ileal segment to reduce the time of contact between urine and mucosa. The result for this patient has been successful and gratifying; renal function studies and electrolytes have been normal without medication for more than six months.

\section{Summary}

In a thirteen-year-old girl profound hyperchloremic acidosis and hypokalemic paralysis developed soon after cutaneous ileocystostomy. Suprapubic cystostomy aided in rapidly correcting the metabolic derangements and provided a unique opportunity to study the pathogenesis of this syndrome in vivo. Rapid absorption of urea and chloride produced a sustained osmotic diuresis. Continued potassium loss was secondary 
to this diuresis, and no active secretion of potassium by the ileum was demonstrated. The ileum also increased urinary sodium concentration which contributed further to electrolyte depletion. Renal handling of acid was normal, but homeostasis was overwhelmed by ileal loop bicarbonate secretion and acidosis occurred.

An attempt at medical therapy was unsuccessful in altering the underlying pathogenetic mechanisms, and surgical intervention was necessary. Since the ileal segment was shortened, the patient has continued to do well; electrolyte and renal function studies have been nearly normal.

C5534 Outpatient Building University Hospital Ann Arbor, Michigan 48104

References

1. Straffon, R. A., and Coppridge, A. J.: Respiratory paralysis and severe potassium depletion after ureterosigmoidostomy, J.A.M.A. 171: 139 (1959).
2. Giesy, J. D., and Hodces, C. V.: Flaccid paralysis associated with hyperchloremic acidosis and hypokalemia following ileal loop urinary diversion, I. Crol 94: 243 (1965).

3. Creevy, C. D, Renal complications after ileac diversion of the urine in non-neoplastic disorders, ibid 83: 394 (1966).

4. LAPIDEs, I.: Mechanism of electrolyte imbalance following ureterosigmoid transplantation, Surg. Cynecol. Obstet. 93: 691 (1951)

5. KAFETSIOUlis, A., and SwINNEY, J.: Urinary diversion by ileal conduit, Br. J. Urol. 40: J (1968)

6. STAmex, T. A.: The pathogenesis and implications of the electrolyte imbalance in ureterosigmoiclostomy. Surg. Gynecol. Obstet. 103: 736 (1956).

7. Anseli. I. S. Geist, R. W., and Creery, (: 1). Estimation of total body potassium in patients with ureterosigmoidostomies, Surg. Gvnecol. Obstet. 112: $322(1961)$.

8. Madsen. P. O.: The etiology of hyperchloremic acidosis following urointestinal anastomosis: an experimental study, J. Urol. 92: 448 (1964).

9. Anslow, W. P., and Wesson, L. G.: Elfect of sus. tained, graded urea diuresis on water and electrolyte excretion, Am. J. Physiol. 180: 605 (1955). 